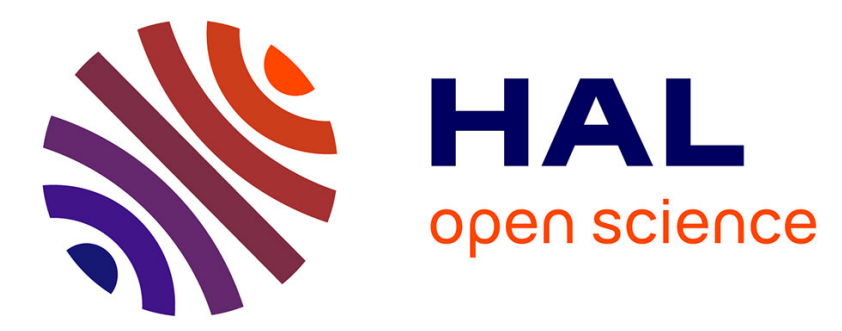

\title{
Value-Added Services, Virtual Enterprises and Data Spaces Inspired Enterprise Architecture for Smart Cities
}

Sobah Abbas Petersen, Zohreh Pourzolfaghar, Iyas Alloush, Dirk Ahlers, John Krogstie, Markus Helfert

\section{- To cite this version:}

Sobah Abbas Petersen, Zohreh Pourzolfaghar, Iyas Alloush, Dirk Ahlers, John Krogstie, et al.. ValueAdded Services, Virtual Enterprises and Data Spaces Inspired Enterprise Architecture for Smart Cities. 20th Working Conference on Virtual Enterprises (PRO-VE), Sep 2019, Turin, Italy. pp.393402, 10.1007/978-3-030-28464-0_34 . hal-02478782

\section{HAL Id: hal-02478782 \\ https://hal.inria.fr/hal-02478782}

Submitted on 14 Feb 2020

HAL is a multi-disciplinary open access archive for the deposit and dissemination of scientific research documents, whether they are published or not. The documents may come from teaching and research institutions in France or abroad, or from public or private research centers.
L'archive ouverte pluridisciplinaire HAL, est destinée au dépôt et à la diffusion de documents scientifiques de niveau recherche, publiés ou non, émanant des établissements d'enseignement et de recherche français ou étrangers, des laboratoires publics ou privés. 


\title{
Value-Added Services, Virtual Enterprises and Data Spaces inspired Enterprise Architecture for Smart Cities
}

\author{
Sobah Abbas Petersen ${ }^{1}$, Zohreh Pourzolfaghar ${ }^{2}$, Iyas Alloush², Dirk Ahlers ${ }^{3}$, \\ John Krogstie ${ }^{1}$ and Markus Helfert ${ }^{2}$ \\ ${ }^{1}$ Dept. of Computer Science, Norwegian University of Science and Technology, Norway \\ ${ }^{2}$ Lero - The Irish Software Research Centre, School of Computing, Dublin City \\ University, Dublin, Ireland \\ ${ }^{3}$ Dept. of Architecture \& Planning, Norwegian University of Science and \\ Technology, Norway \\ Email: \{sap, krogstie, Dirk.ahlers\}@ntnu.no; \\ \{markus.helfert, Zohreh.Pourzolfaghar, iyas.alloush\}@dcu.ie
}

\begin{abstract}
As a part of their digital transformation, municipalities across Europe have taken initiatives to support Open Data platforms and provide services leveraging on data. This challenges the traditional business driven IT strategy promoted by several Enterprise Architecture methodologies, which are designed to operate within a single enterprise that has a complete overview of its data and ICT systems. We envisage scenarios where public and private collaborative networks provide value added services to its citizens by leveraging on data. In this paper, we propose an Enterprise Architecture Framework for Cities to support them maneuvre smartly within their data space to create value added services through a variety of collaborative networks or Virtual Enterprises that bridge organisational boundaries. The novel elements of this Enterprise Architecture Framework are a DataxChange, the Value-Added Services and Virtual Enterprise layers. This work has been conducted within the EU H2020 Smart City project +CityxChange.
\end{abstract}

Keywords: Data Spaces, Enterprise Architecture Framework, Smart Cities, Collaborative Networks, Positive Energy Blocks.

\section{Introduction}

Sustainability and digital transformation and are two of the main driving forces for cities and municipalities today. Current Smart City approaches have advanced from monitoring various aspects of a city through sensor and other data to providing services to its citizens in more collaborative ways [1,2]. We adopt the view of a smart city that integrates people, technology and information to create a sustainable and resilient infrastructure that provides high quality services for its citizens [3]. The data that is gathered through sensors and/or other sources are central to developing value added services for the citizens. We envisage scenarios where public and private 
collaborative networks (CNs) provide value added services to its citizens by leveraging on data and systems that bridge different organisations.

The work reported in this paper is motivated the EU H2020 project + CityxChange, which focuses on Positive Energy Blocks (PEBs) to reduce emissions in cities and communities towards reaching the Paris Climate Goals [4]. Taking a broad perspective of cities and communities in the light of UN's Sustainable Development Goals (SDG); in particular SDGs 7, 11 and 13 (Affordable Clean Energy, Sustainable Cities and Societies and Climate action respectively), there is a need to engage citizens and to develop a portfolio of value added services that will enable citizens to increase their awareness about environmental sustainability and to help them make better choices. Furthermore, cities are motivated to engage their private businesses and other stakeholders in novel and innovative partnerships to provide value added services to the citizens.

One of challenges faced by cities and service providers is the lack of access to relevant data or indeed an overview of available data and relevant actors. Enterprise Architecture (EA) used initially in the business world to bridge between the business needs and the ICT strategy of a company has been used in Smart City contexts to bridge the needs of a city with ICT, resulting in a number of Smart City Enterprise Architecture Frameworks (EAFs). Our review of several EAFs from Smart City projects show that while several of them identify the need for services, the emphasis on the role of data is not so evident. We argue that the role of data must be a central component of a Smart City EAF, to support the drive for digital transformation as well as the effective use of available data.

In this paper, we propose a layered EAF for + CityxChange and Smart Cities in general where a DataxChange lies at the heart of the EAF. The DataxChange bridges the technology layers and the higher layers of the architecture, such as the business collaborations and value added services. The proposed architecture builds upon existing work, and complements them by emphasising the novel elements, which are the DataxChange, the Value-Added Services and the Virtual Enterprise (VE) layers to support CNs.

This paper is structured as follows: Section 2 describes the +CityxChange project; Section 3 describes our vision for a Smart City EAF; Section 4 describes the + CityxChange EAF to realise such a vision; Section 5 illustrates how value added services can be supported by EAF; Section 6 gives an overview of related work and Section 7 summarises the paper and provides an outline of the future work.

\section{$2+$ CityxChange Project}

The background and motivation for this paper is the EU H2020 funded +CityxChange project [5] that aims to develop PEBs in smart cities and communities as part of emission reductions to reach the Paris Climate Goals [4]. It is a Lighthouse project, where Trondheim, Norway, and Limerick, Ireland, are the lighthouse cities, and five follower cities showcase replication. The consortium of 32 partners include universities, municipalities, utility and infrastructure providers and other private 
entrepreneurs. It follows an integrative approach with a strong focus on city integration, open innovation and replicability. The approach combines:

- Integrated Planning and Design of cities;

- Creation and Enabling of a Common Energy Market;

- A CommunityxChange with all stakeholders of the city to create connected and engaged communities.

The project focuses on the technical solutions as well as the interactions and integration between buildings, users, cities and the energy system. It also focuses on the implications and impact on city planning, city systems, energy trading, citizen involvement, policies and regulations, data and digitalisation and socio-economic issues. A Positive Energy Blocks (PEB) is defined by the EU as several buildings that actively manage their energy consumption and the energy flow between them and the wider energy system ${ }^{1}$. They achieve an annual positive energy balance through use, optimization and integration of advanced materials, energy reduction, local renewable energy production and storage, smart energy grids, demand-response, energy management of electricity, heating and cooling, user involvement. PEBs or Positive Energy Districts (PEDs) are designed as an integral part of the district energy system. They should be intrinsically scalable up to positive energy districts and cities and are well embedded in the spatial, economic, technical, environmental and social contexts.

New forms of integrated spatial, social, political, economic, regulatory, legal and technological innovations are envisaged to deliver citizen observatories, innovation playgrounds and regulatory sandboxes linked to Urban Living Labs. Municipalities will develop Bold City Visions to engage civil society, local authorities, industry and Research and Technology Organisations to scale up from PEBs to PEDs or Positive Energy Cities, supported by a distributed and modular energy system architecture.

The ICT related challenges faced by the + CityxChange project include the lack of a means or forum for discovering existing and available data and services and indeed accessing and retrieving relevant data from available data sources [6], for use in developing value added services. ICT is envisaged to play a central role as an enabler for innovative value added services, stimulating new collaborations and in achieving PEBs and scaling up to the district and city levels. Similarly, ICT will provide support for the replication of solutions from the Lighthouse cities to the follower cities $[4,6]$.

\section{Vision}

Our vision for an EAF to support the +CityxChange project and Smart Cities in general is driven by the need and availability of data and systems and the central role that data plays in today's cities and how the data could be leveraged to provide valueadded services to the citizens. Many Smart City concepts are inherently multidisciplinary and need the collaboration of various stakeholders from different domains, including cities and citizens [7]. New and innovative value added services could be provided by diverse constellations of CNs or VEs, which are a group of

${ }^{1}$ https://ec.europa.eu/info/funding-tenders/opportunities/portal/screen/opportunities/topicdetails/lc-sc3-scc-1-2018-2019-2020 
partners that collaborate to achieve a specific goal [8]. Our vision will address some of the shortcoming of existing EAFs to meet the needs of smart cities.

We believe that data can play a central role in bridging the technology aspects to the strategic and business aspects of an enterprise, such as the business collaborations and value added services. The central role of data and its value to an organisation has been discussed in many contexts, such as Industry 4.0 [9]. The need for supporting collaborative data analysis and processing and enhancing collaboration support among the data owners, data scientists and indeed service providers have received attention [10]. Examples of leveraging on data in the context of smart cities have appeared recently in the literature; in Helsinki, the city has been considered as a Living Lab and the data that is collected has been used to develop services as mobile applications [11]. The need for easy discovery of existing and available data and services and indeed accessing and retrieving relevant data is increasing and is one of the main challenges in the +CityxChange project. Thus, an EAF that supports an overview of data and the meta-data related will be an important asset to the creation and operation of value-added services in a smart city. If the stakeholders of a smart city have an overview of data that is potentially accessible and available for them to leverage on, the potential of value added services for the citizens can be enhanced.

CNs and VEs have long been discussed as means and drivers for value creation [12]. More recently, they have been discussed as an important mechanism in the context of leveraging on the vast amounts of data that are available in many contexts; e.g. [13]. Bringing these concepts to the heart of an EAF is essential in supporting the creation of innovative and value added services in the context of smart cities. This can facilitate the integration of services vertically within a city such as technological support, data processing as well as operational services, and connecting them to a clearly identified value to the city and citizens. Similarly, it can ensure the integration of the processes horizontally across the value chain around data, such as the data owners, processors and data consumers. Furthermore, this can support empowerment not only of the citizens, but provide opportunities for project partners to collaborate in new and innovative CNs, VEs and business models.

\section{4 +CityxChange Enterprise Architecture Framework}

Based on the literature and the smart city EAFs developed in relevant EU projects, such as the Smart City project SmartEnCity [2] and the standardisation efforts in ESPRESSO [1], we propose a layered EAF for the +CityxChange project, as illustrated in Figure 1. The main driver for the EA is the "service-based ecosystem", identified as an objective for the EA in the +CityxChange project [6]. The scenarios envisaged for the project span many application domains (or silos) such as energy, transport, IoT, built environment and governance. An example of a value added service considered in the project that spans several application domains is eMobility as a service, across energy, transport and urban planning domain.

Several horizontal layers are defined in the EAF to support value added services, which utilise data and are provided by collaborations among two or more enterprises; i.e. by a VE. The thinking behind the layered approach is that each layer supports the 
layer above it. Thus, the value-added services are supported by one or more businesses or a VE, which are supported by one or many applications, which use data from the + CityxChange DataxChange layer. The +CityxChange DataxChange is supported by technologies and finally, the data is obtained from data sources.

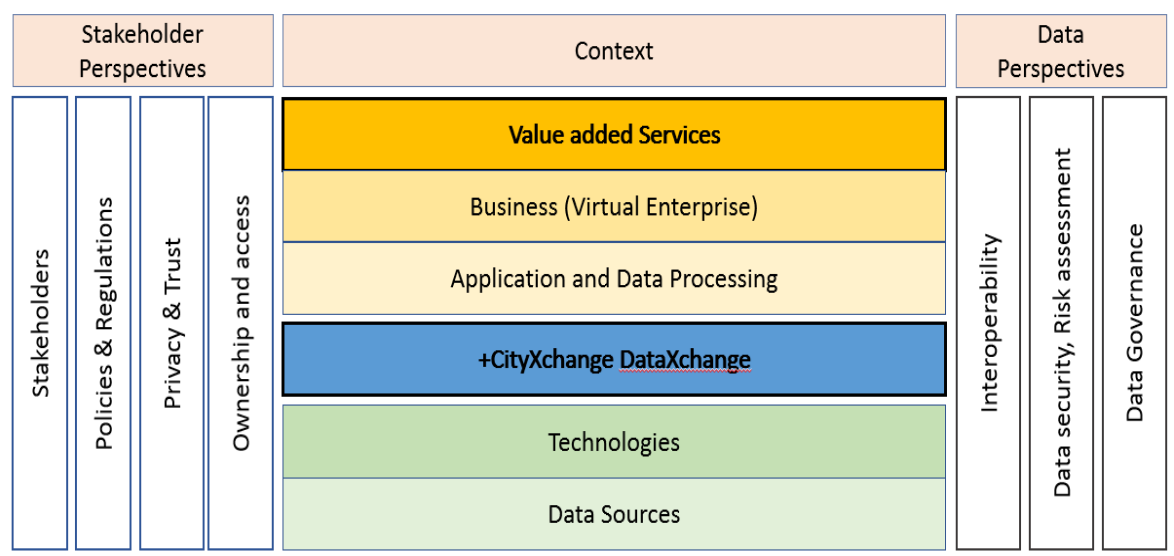

Figure $1+$ CityxChange Enterprise Architecture Framework

The +CityxChange DataxChange is at the heart of the EAF, where this layer provides an overview of all available data and systems and relevant information about the data that is required for creating value added services. Relevant information about the data will include the owner of the data, how the data may be accessed and the conditions and criteria that may be necessary to access or use the data. The DataXchange plays a central role is providing a comprehensive overview of the available data, providing the support that the various service providers need. Indeed, it should provide information about the relevant and potential collaboration partners that could participate in providing value added services to citizens, leveraging the data. The DataXchange provides a means to bridge the lower levels of the EAF, such as supporting technologies, and the higher levels of the EAF, such as the value added services. Furthermore, this layer will also serve as a common "platform" for the lower layers to facilitate access to data for the higher layers. Together with the Business Layer, this layer is designed to support concepts such as data markets and data as a commodity in the near future.

The highest layer of the EA is the context layer, which drives the services and the business context. Value added services are created to meet the needs of the citizens or to affect and change their behaviour to meet the strategy of the city or some business actor. The Value added Service layer is below the Context layer, which both offers services to and uses data and applications from the different application domains and involves one or more actors. The Application and Data Processing Layer describes the different applications that support the services. Applications may support processing or analytics of data that are relevant for the service or other purposes such as searching and navigating the information space, visualisation of information or other.

Two layers are defined below the DataXchange: Technologies layer and Data Sources layer. The Technologies layer describes the different technologies that 
support the DataXchange and the higher layers of the EAF. Technologies include hardware (e.g. servers and sensors, metering devices), applications such as databases and infrastructure, e.g. for micro payments. As data is obtained from a variety of sources such as IoT, sensors or local renewable energy production such solar panels mounted on private property, the origin of the data is essential for data governance; for ensuring the quality of the data, fostering trust among the users of the data, for supporting transparency and integrity of data, and for adhering to regulatory frameworks such as GDPR. Thus the Data sources layer is important to identify the sources that provide the data.

Related to the horizontal layers, we have two perspectives: (i) Stakeholder perspective to highlight the different stakeholders, their roles and perspectives; and (ii) Data perspective to address the specific principles and guidelines that are relevant in data-rich environments. These perspectives apply to all or most levels of the EAF as shown in Figure 1. A number of high level principles were identified for the + CityxChange EAF in [6]. These perspectives provide a means of structuring and contextualising guidelines and principles for using the +CityxChange EAF. A detailed description of principles and guidelines are provided in [14].

\subsection{Stakeholder Perspectives}

The +CityxChange project has a citizen focussed approach with several tasks dedicated to citizen engagement. Similarly, the service-based ecosystem and the participation of several public and private entities call for close attention to the diverse stakeholders that are a part of the city-wide or +CityxChange-wide EA space. Stakeholders describe the various entities involved in the city-wide EA space, which include citizens, service providers and consumers, who develop service to create added value. Stakeholders also include data owners, providers, processors and consumers of data, public authorities, private enterprises, researchers and various communities (e.g. a local housing complex).

Privacy and Trust describes the relevant principles and guidelines that need to be followed not only to respect and protect the privacy of individual people and organisations, but also support a network of trust among the various stakeholders of the +CityxChange DataxChange and value added services. Given the focus on stakeholders and the protection of individuals through the recent EU GDRP regulations, we have chosen to separate privacy and trust from data security (although they are dependent on one another). An EAF for a smart city spans beyond organisational boundaries and consequently the potential of sharing privacy-related data among stakeholders and data processors from a number of different prganisations can be envisaged. Thus, the need to increase awareness of privacy-related issues and designing to ensure privacy and trust and compliance to regulations, in particular with respect to the use of stored data for multiple purposes, increases.

Policies and regulations describe the regulatory stakeholders that are involved in the project, the influencers and stakeholders and services that are affected by the specific policies and regulations. Ownership and access describe the relationships between the stakeholders and the entities that will be represented in the layers of the 
EAF. The scenarios envisaged in the project highlight that the relationships between the data and other entities or the ownership or accessibility models for data elements are not simple, uniform or trivial.

\subsection{Data Perspectives}

The + CityxChange project envisages value added services that leverage on data and system integration that is available through the +CityxChange DataxChange or indeed Open Data. Thus, there is a data perspective related to all layers of the EAF, from the data sources to the value added services. This raises a need to address the data perspectives.

Interoperability addresses how entities, through all the layers in the EAF, could be brought together in a cohesive way to provide the value added services. Interoperability applies to all the levels of the EAF.

Data security and risk assessment apply to all the layers of the EAF to ensure that the data is handled in a secure and reliable manner. The security of data is not a static state; i.e. data that is secure at any time may not be secure at a future time, and thus requires regular assessments of risks and threats to the security of the data.

Data Governance ensures proper data management and ownership processes and data quality and encompasses people, organisations and processes. Data governance ensures that data is consistent, available and useable.

\section{$5 \quad$ Value Added Services}

One or more value added services (example services A, B and X) could be created by using the data available from the +CityxChange DataxChange, as shown in Figure 2.

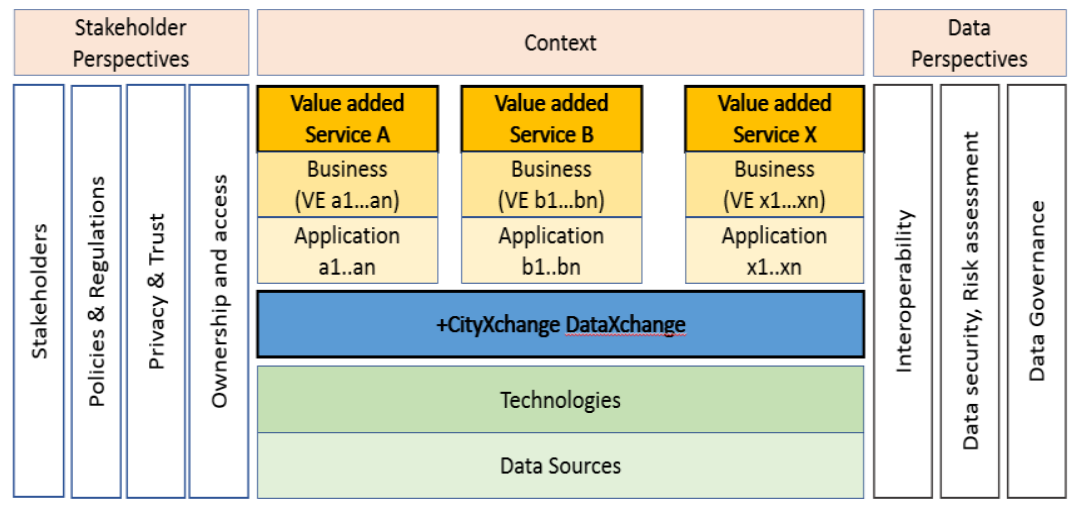

Figure 2 Value added Services as an instance in the + CityxChange EAF

Each of the services are likely delivered by collaboration among one or more organisations; any organisation may participate in the delivery of one or more services and participate in one or more VEs. For example, the municipality of a 
specific city may be a part of a VE that provides both the Services A and B. Similarly, the same municipality may also play a role in Service X, perhaps different to that for the Services A and B. This simple example illustrates that several elements (data/services) from the DataxChange may be used by one or more services.

Based on the scenarios envisaged for the +CityxChange project and the visions of the Lighthouse cities that have been shared and integrated so far, we see the need to support such value added services and the need to share data and the ability to incorporate new data that is generated through the value added services. The + CityxChange DataxChange is designed to facilitate such activities.

\section{Related Work}

EA concerns the overall ICT landscape and alignment of the business strategy and its ICT implementation. Several EAFs have been proposed for Smart Cities. Based on the leading EAF in the industry [15], The Open Group's EA, TOGAF [16], several Smart City EAFs are based on a multi-tier or layered approach proposed; e.g. [1, 1720]. The notion of services is identified explicitly in some of the EAFs $[1,2,19,21]$. The service layer identifies the business and the stakeholder perspectives, often considered as services offered by municipalities or the cities.

Of particular interest to our work is the Intelligent Data Spaces Reference Architecture Model (IDS-RAM), proposed by the German Fraunhofer Institute and the International Data Spaces Initiative [9]. They describe an Industrial Data Space as a "virtual data space leveraging existing standards and technologies as well as accepted governance models for the data economy, to facilitate the secure and standardized exchange and easy linkage of data in a trusted business ecosystem". Similar to other EAFs, IDS-RAM also takes a layered approach. However, a few things distinguish it from the other frameworks and methodologies; e.g. it focuses on the Industrial Data Space and it takes a data-centric approach and thus supports both top-down and bottom-up approaches. A framework which describes foundation and principles for Information Technology in smarter cities is proposed in [22]. It incorporates instrumented data from sensors, Integration of data and intelligent inclusion of complex analytics, modelling and optimization, and visualization in operational business processes.

\section{Summary and Future Work}

An EAF to support a service-based ecosystem for the + CitxChange project is proposed, which supports the creation of value added services that leverage available data and services/systems. The novel elements of this Enterprise Architecture are the DataxChange, the Value-Added Services and the Virtual Enterprise layers. A DataxChange is at the heart of this architecture, which we argue is an essential part of a Smart City EAF, and which will bring together all available data and relevant information about the data to support the creation of value added services, by collaborative networks of partners from public private and research sectors. The 
DataXchange layer provides a bridge between the lower levels of the EAF, such as supporting technologies and data sources, and the higher levels of the EAF, such as the value added services.

The proposed architecture is work in progress and we are working on refining this EAF by applying it to describe the different services that will be developed in the project. Furthermore, this EAF is envisaged as a support in reaching out to the partners who will participate in the VEs providing the value added services, and in driving the dialogue in the iterative definitions of the value added services. As such, it can also support many of the collaborative relationships within a complex project and provide additional technical frameworks and collaboration spaces, based on a joint understanding of the ICT relationships and networks within the project. These should also support easier communication, information access, and knowledge finding and sharing within the project. Our next steps are to describe a few scenarios and services using this EAF and facilitate the service description process, while refining the EAF based on the feedback.

\section{Acknowledgements}

+ CityxChange is a smart city project under the Smart Cities and Communities topic that is funded by the European Union's Horizon 2020 Research and Innovation programme under Grant Agreement No. 824260. The authors would like to thank the project partners from Trondheim Municipality, Limerick City Council and the participants of work packages 1 and 2 as well as the whole project team.

\section{References}

[1] ESPRESSO project (systEmic Standardization apPRoach to Empower Smart citieS and cOmmunities)), Deliverable D4.2 - Definition of Smart City Reference Architecture. 2016.

[2] SmartEnCity, Deliverable 6.3: Data Model Architecture Implementation. 2017.

[3] N. Villanueva-Rosales, R.L. Cheu, A. Gates, N. Rivera, O. Mondragon, S. Cabrera, and C. Ferregut, A collaborative, interdisciplinary initiative for a smart cities innovation network, in EEE First International Smart Cities Conference (ISC2). 2015, IEEE: Guadalajara, Mexico. p. 25-28 Oct.

[4] United Nations Climate Change. What is the Paris Agreement? [cited 201920 May]; Available from: https://unfccc.int/process-and-meetings/the-paris-agreement/what-isthe-paris-agreement

[5] +CityXChange. Positive Energy Blocks. 2019 [cited 201919 May]; Available from: http://cityxchange.eu/.

[6] D. Ahlers, L.W.M. Wienhofen, S.A. Petersen, and M. Anvaari, A Smart City Ecosystem enabling Open Innovation, in 19th International Conference Innovations for Community Services (I4CS). 2019: Wolfsburg, Germany.

[7] D. Ahlers, P. Driscoll, E. Löfström, J. Krogstie, and A. Wyckmans, Understanding Smart Cities as Social Machines, in Workshop on the Theory and Practice of Social Machines @ WWW2016, IW3C2, 2016. 2016. 
[8] S.A. Petersen, Virtual Enterprise Formation and Partner Selection: An Analysis Using Case Studies. International Journal of Networking and Virtual Organisations, 2007 4(2): p. 201-215.

[9] B. Otto, S. Lohmann, S. Steinbuß, and A. Teuscher, IDS Reference Architecture Model, Industrial Data Space, Veriosn 2.0. 2018, International Data Spaces Association \& Fraunhofer.

[10] K. Park, M.C. Nguyen, and H. Won, Web-based collaborative big data analytics on big data as a service platform, in 17th International Conference on Advanced Communication Technology (ICACT). 2015 IEEE.

[11] H. Hielkem and P. Hongisto, Developing the Helsinki Smart City: The Role of Competitions for Open Data Applications. Journal of the Knowledge Economy, 2013. 4(2): p. 190-204.

[12] L. Camarinha-Matos and H. Afsarmanesh, COLLABORATIVE NETWORKS- Value creation in a knowledge society, in: Knowledge Enterprise: Intelligent Strategies in Product Design, Manufacturing, and Management. PROLAMAT 2006, K. Wang, G.L. Kovacs, W. M., and F. M., Editors. 2006, Springer: Boston, MA.

[13] L. Camarinha-Matos, R. Fornasiero, and H. Afsarmanesh, Collaborative Networks as a Core Enabler of Industry 4.0, in: Collaboration in a Data-Rich World. PRO-VE 2017, L. Camarinha-Matos, R. Fornasiero, and H. Afsarmanesh, Editors. 2017, Springer, Cham. p. 3-17.

[14] S.A. Petersen, I. Alloush, Z. Pourzolfaghar, and M. Helfert, +CityxChange - D1.2 Report on the Architecture for the ICT Ecosystem - Initial Version. 2019.

[15] B.H. Cameron and E. McMillan, Analyzing the current trends in Enterprise Architecture Frameworks. Journal of Enterprise Architecture, 2013. 9(1): p. 60-71.

[16] The Open Group, The Open Group Architecture Framework TOGAF Version 9.1. 2011.

[17] C. Harrison and I.A. Donnelly, A Theory of Smart Citie, in Proceedings of the 55th Annual Meeting of the ISSS-2011. 2011: Hull.

[18] IEC International Electrotechnical Commission. ISO/IEC JTC 1 Information technology, WG11 on Smart Cities. 2019 [cited 201929 April]; Available from: https://www.iec.ch/dyn/www/f?p=103:14:34897880612264:..:FSP ORG ID,FSP LAN G ID: 12973,25 .

[19] Z. Pourzolfaghar, V. Bastidas, and M. Helfert, Standardisation of Enterprise Architecture Development for Smart Cities. Journal of Knowledge Economy (under publication), 2019.

[20] R. Winter and R. Fischer, Essential Layers, Artifacts, and Dependencies of Enterprise Architecture. Journal of Enterprise Architecture, 2007.

[21] D. Ferguson, J. Sairamesh, and S. Feldman, Open Frameworks for Information Cities. Communications of ACM, 2004. 47(2): p. 45-49.

[22] C. Harrison, B. Eckman, R. Hamilton, P. Hartswick, J. Kalagnanam, Paraszczak, and P. Williams, Foundations for Smarter cities. IBM J. RES. \& DEV, 2010. 54(4): p. 1-16. 